\title{
Pain over speed bumps in diagnosis of acute appendicitis: diagnostic accuracy study
}

\author{
(C) $(1) \Theta$ OPEN ACCESS
}

\author{
Helen F Ashdown academic clinical fellow in general practice ${ }^{1}$, Nigel D'Souza specialist registrar \\ in general surgery ${ }^{2}$, Diallah Karim foundation traine ${ }^{2}$, Richard J Stevens senior medical statistician ${ }^{1}$, \\ Andrew Huang consultant colorectal and general surgeon ${ }^{2}$, Anthony Harnden university lecturer in \\ general practice $^{1}$
}

${ }^{1}$ Department of Primary Care Health Sciences, University of Oxford, Oxford OX2 6GG, UK; ${ }^{2}$ Department of Surgery, Stoke Mandeville Hospital, Aylesbury HP21 8AL, UK

\begin{abstract}
Objective To assess the diagnostic accuracy of pain on travelling over speed bumps for the diagnosis of acute appendicitis.

Design Prospective questionnaire based diagnostic accuracy study.

Setting Secondary care surgical assessment unit at a district general hospital in the UK.

Participants 101 patients aged 17-76 years referred to the on-call surgical team for assessment of possible appendicitis.

Main outcome measures Sensitivity, specificity, positive and negative predictive values, and positive and negative likelihood ratios for pain over speed bumps in diagnosing appendicitis, with histological diagnosis of appendicitis as the reference standard.

Results The analysis included 64 participants who had travelled over speed bumps on their journey to hospital. Of these, 34 had a confirmed histological diagnosis of appendicitis, 33 of whom reported increased pain over speed bumps. The sensitivity was $97 \%$ (95\% confidence interval $85 \%$ to $100 \%$ ), and the specificity was $30 \%$ (15\% to $49 \%$ ). The positive predictive value was $61 \%$ ( $47 \%$ to $74 \%)$, and the negative predictive value was $90 \%$ ( $56 \%$ to $100 \%$ ). The likelihood ratios were 1.4 (1.1 to 1.8 ) for a positive test result and 0.1 ( 0.0 to 0.7$)$ for a negative result. Speed bumps had a better sensitivity and negative likelihood ratio than did other clinical features assessed, including migration of pain and rebound tenderness.
\end{abstract}

Conclusions Presence of pain while travelling over speed bumps was associated with an increased likelihood of acute appendicitis. As a diagnostic variable, it compared favourably with other features commonly used in clinical assessment. Asking about speed bumps may contribute to clinical assessment and could be useful in telephone assessment of patients.

\section{Introduction}

Speed bumps are a commonly used traffic calming device to reduce the speed of vehicles. ${ }^{1}$ Although controversial, traffic calming measures have been associated with a $70 \%$ decrease in injuries among child pedestrians in some areas, ${ }^{2}$ and they may be a promising intervention for reducing the overall number of road traffic injuries and deaths. ${ }^{3}$ However, speed bumps may have a useful alternative benefit in the diagnosis of acute appendicitis.

Acute appendicitis is the most common surgical abdominal emergency. ${ }^{4}$ Rapid diagnosis is important, because increased time between onset of symptoms and surgical intervention is associated with increased risk of appendiceal perforation and therefore potential peritonitis, sepsis, and death. ${ }^{5}$ However, the rate of negative appendicectomy (when appendicectomy is performed, but the appendix is found to be normal on histological evaluation ${ }^{4}$ ) ranges from $5 \%$ to $42 \%,{ }^{6}$ and this can be associated with considerable morbidity. ${ }^{7}$ Clinical diagnosis can be challenging, particularly in the early stages of appendicitis when clinical manifestations may be quite non-specific or atypical. Different elements of history, examination, and laboratory findings have varying predictive power in the diagnosis of appendicitis, ${ }^{6}$ and algorithms and scoring systems for clinical evaluation exist, ${ }^{4}$ but appendicitis can nevertheless be easily missed. ${ }^{8}$ 
Patients with appendicitis have sometimes been noted to complain of a worsening of their abdominal pain when they travel over speed bumps. $\Downarrow$ Some doctors ask about this routinely as part of history taking, believing it to be a highly diagnostic feature (personal communication). We sought to determine whether any evidence supports this practice and to determine its predictive power as a diagnostic sign.

\section{Methods}

We did a prospective study at a district general hospital in Buckinghamshire in the United Kingdom. Roads in the county of Buckinghamshire are almost universally surfaced in tarmac and are smooth, with any speed bumps raised from the road surface in a variety of designs and elevations. All patients aged 16 or over who had been referred to the on-call surgical team as part of their usual care, by either a general practitioner or an emergency department doctor, with suspected appendicitis were eligible. They were identified consecutively over a six month period between February and August 2012.

We asked participants to complete a questionnaire survey about their symptoms, including four specific questions related to their journey into hospital: mode of transport, whether they had travelled over speed bumps, whether they had had pain on the journey, and whether the pain changed when they went over a speed bump. We defined patients as "speed bump positive" if they had a worsening of pain from baseline over speed bumps and as "speed bump negative" if their pain stayed the same, if they were unsure, or if their pain improved on going over speed bumps. To minimise recall bias, patients had to complete the questionnaire within 24 hours of arrival in hospital and before they had been to theatre. We also recorded examination findings on admission from their notes. Two of the authors entered data on to a spreadsheet, and a third author double checked transcription.

We then followed participants through their admission to determine the outcome and whether they were taken to theatre for presumed appendicitis. For those who had been to theatre, we obtained the subsequent histology report. We used histological diagnosis of appendicitis as the reference standard, which is the usual practice in studies of appendicitis. ${ }^{6}$ One of the authors, who was blinded to all clinical details of the participants, corroborated interpretation of the histology findings. We also asked participants to provide contact details so that, if an alternative diagnosis or no diagnosis was made, we could contact them after their admission to ensure that their symptoms had resolved, to avoid missing cases of subacute or "grumbling" appendicitis. A positive or negative histological diagnosis of appendicitis was made in participants who went to theatre and had their appendix removed. We assumed participants whose symptoms resolved without surgery to have a negative diagnosis. We confirmed resolution of symptoms by telephone follow-up between two weeks and three months after admission.

In pilot data (11 cases and 21 controls) collected in 2009, the sensitivity was $82 \%$ (95\% confidence interval $48 \%$ to $98 \%$ ) and the specificity was $67 \%$ ( $43 \%$ to $85 \%$ ). We used the R software package to simulate studies of varying sizes on the basis of these estimates. We calculated that $100-150$ participants in the main study would be sufficient to show a likelihood ratio greater than 1.8-2.0.

We calculated the sensitivity, specificity, positive and negative predictive values, and positive and negative likelihood ratios, with $95 \%$ confidence intervals, for the outcome diagnosis of appendicitis. When a sign was recorded as "unsure," we considered it absent for the purposes of calculation. We restricted the primary analyses to those patients reported to have travelled over speed bumps on the route to the hospital. We also planned to compare the diagnostic accuracy of worsened pain over speed bumps with more conventional diagnostic features of appendicitis, such as migratory pain and rebound tenderness. We used the "diagt" command in Stata (Release 11) for calculations.

\section{Results}

One hundred and one patients were recruited into the study. The median age was 34 (range 17-76) years. Sixty one participants were taken to theatre for presumed appendicitis, of whom 54 had their appendix removed. Acute appendicitis was confirmed histologically in 43 of these, giving a negative appendicectomy rate of $20 \%$.

Sixty eight participants had travelled over speed bumps. We excluded four patients from diagnostic accuracy analysis: one because histology was not available, and three because they were treated with antibiotics as an alternative to surgery, so diagnosis was not confirmed histologically. Of the 64 patients in the main analysis, 31 were recruited between 9 am and $5 \mathrm{pm}$, 24 between $5 \mathrm{pm}$ and $10 \mathrm{pm}$, and nine between $10 \mathrm{pm}$ and 9 am. Fifty eight patients travelled to the hospital by car and six by ambulance, of whom five had pain over speed bumps and a final diagnosis of appendicitis and one had no pain over speed bumps and no appendicitis.

Table $1 \Downarrow$ shows pain over speed bumps in relation to diagnosis of appendicitis. Fifty four of 64 participants were "speed bump positive." Thirty four participants had a confirmed diagnosis of appendicitis, 33 of whom had worsened pain over speed bumps, giving a sensitivity of $97 \%$ ( $85 \%$ to $100 \%)$ and a specificity of $30 \%$ (15\% to $49 \%$ ). The positive predictive value was $61 \%$ ( $47 \%$ to $74 \%)$, and the negative predictive value was $90 \%(56 \%$ to $100 \%)$. The likelihood ratios were 1.4 (1.1 to 1.8$)$ for a positive test result and 0.1 (0.0 to 0.7$)$ for a negative result. Table $2 \Downarrow$ shows how this compares with other clinical variables commonly used for diagnosis of appendicitis and also assessed in our sample.

Seven patients who were "speed bump positive" but did not have appendicitis had other important abdominal diagnoses, such as a ruptured ovarian cyst or diverticulitis. A post hoc secondary analysis of the diagnostic accuracy of pain over speed bumps for the diagnosis of important abdominal pathology requiring treatment (including appendicitis) increased the sensitivity to $98 \%$ (87\% to $100 \%$ ) and the specificity to $39 \%$ (20\% to $61 \%)$.

Thirty three patients did not recall having travelled over speed bumps. A sensitivity analysis classifying those patients who did not recall travelling over speed bumps as having no pain over speed bumps had the effect of decreasing the sensitivity to $77 \%$ (61\% to $88 \%$ ) and increasing the specificity to $61 \%$ (47\% to $74 \%$ ), with a positive likelihood ratio of 2.0 (1.4 to 2.9) and a negative likelihood ratio of 0.4 (0.2 to 0.7 ) (see web extra data).

\section{Discussion}

Our results confirm that an increase in pain while travelling over speed bumps is associated with an increased likelihood of acute appendicitis. Absence of pain over speed bumps is associated with a significantly decreased likelihood of appendicitis. Although the specificity was relatively low, as a diagnostic variable pain over speed bumps compared favourably with other features commonly used in diagnostic assessment, 
with a better sensitivity and negative likelihood ratio than all other features assessed. Moreover, some patients who were "speed bump positive" but did not have appendicitis had other important abdominal diagnoses, such as a ruptured ovarian cyst, diverticulitis, or pelvic inflammatory disease. We hypothesise that the worsening of pain when travelling over speed bumps in appendicitis may occur because the movement involved irritates the peritoneum in a similar way to that produced by testing for rebound tenderness on examination.

\section{Strengths and limitations of study}

Strengths of our study include the standardised approach to gathering information from patients by using a questionnaire and the obtaining of this information early in their admission and thus soon after their journey. A potential weakness is that although we recruited 101 patients as planned from our sample size calculation, only 68 recalled having travelled over speed bumps, a much lower rate than in our pilot study, which may be related to a redevelopment of the hospital site. Because of this, the number used for analysis (64 patients) was less than planned, leading to moderately large confidence intervals.

The presence of pain over speed bumps may have been overestimated in some patients owing to recall bias. Patients who had pain over speed bumps would be more likely to recall having travelled over them, whereas those who had no worsening of pain would not necessarily remember them. Although the sensitivity was $97 \%$ (table $2 \Downarrow$ ) for patients who recalled speed bumps, because 33/97 (34\%) patients did not travel (or did not recall travelling) over speed bumps, this diagnostic sign is not available in all patients and would therefore detect $77 \%$ (61\% to $88 \%$ ) of cases of appendicitis. This compares favourably with the other diagnostic features we assessed (see web extra data). Variable exposure to speed bumps would also occur in clinical practice, so ours is a pragmatic study that shows that pain over speed bumps can be a useful diagnostic sign when available, although availability will vary.

We used histological diagnosis of appendicitis as the reference standard for diagnosis. Three patients in our sample were treated with antibiotics for presumed appendicitis while waiting for surgery but went on to make a full recovery. A systematic review published during recruitment to our study has shown that antibiotics can lead to resolution of acute appendicitis. ${ }^{9} \mathrm{We}$ made the decision to exclude these patients from the analysis owing to the lack of a confirmed diagnosis, but a sensitivity analysis including these patients and classifying them in turn as positive or negative for a diagnosis of appendicitis made very little difference to overall results.

\section{Comparison with other studies}

Andersson (2004) did a meta-analysis of the diagnostic accuracy of clinical features of appendicitis. ${ }^{6}$ Our finding of a negative likelihood ratio of 0.1 for pain over speed bumps in the diagnosis of appendicitis outperformed not only other clinical variables in our study (as shown in table $2 \Downarrow$ ) but also those in Andersson's meta-analysis - migratory pain (0.52), nausea or vomiting (0.72), and rebound tenderness (0.39). Our positive likelihood ratio of 1.4 was similar to the findings of the meta-analysis for the above features. Another study, which also investigated the accuracy of various methods of diagnosis in 100 patients with possible appendicitis, found that the symptom of pain due to bumpiness in the road (which they termed the "cat's eye symptom") had a sensitivity of $80 \%$ and a specificity of $52 \%{ }^{10}$ The "cat's eye symptom" had to be volunteered by the patient to be classed as positive, whereas in our study the response to speed bumps was solicited directly in a questionnaire. Our higher sensitivity of $97 \%$ but lower specificity of $30 \%$ may be related to the use of elicited rather than volunteered symptoms, for which one would predict exactly this difference in results.

\section{Conclusions and implications}

The high sensitivity of pain over speed bumps gives it a strong "rule-out value" and makes it a useful tool to use in excluding appendicitis and other important abdominal diagnoses. The low specificity, however, means that many patients with pain over speed bumps will not necessarily have appendicitis (that is, it is a poor "rule-in" test). Potential exists for it to be incorporated into clinical prediction rules for appendicitis. Our study was based in secondary care, so our results are not necessarily generalisable to a primary care population. However, pain over speed bumps could potentially have a useful role in primary care in assisting in the telephone assessment of patients with abdominal pain. As all our group of patients had already been assessed by a clinician who thought they might have appendicitis, the pre-test probability is quite high; the speed bump test might also be useful in assessment of all types of abdominal pain, not just when appendicitis is suspected. A history of pain on travelling over uneven road surfaces or potholes may provide a useful proxy for speed bumps in healthcare settings where speed bumps are less frequently found.

Although being "speed bump negative" offers some reassurance against a diagnosis of appendicitis, being "speed bump positive" certainly does not guarantee a diagnosis of appendicitis, so in this respect the myth is untrue. However, our findings suggest that questioning about speed bumps should form a routine part of the assessment of patients with possible appendicitis.

Unanswered questions include whether the speed or manner of driving approach to a speed bump affects the diagnostic power.

We thank A K Allouni and S Kreckler for their contributions to the original idea for the project and pilot study. We also thank doctors in the surgical team at Stoke Mandeville Hospital for their assistance with recruitment.

Contributors: HFA developed the idea and designed the study, analysed and interpreted the data, and wrote the paper. ND'S and DK participated in study design, collected the data, and helped to write the paper. RJS assisted with study design, analysed and interpreted the data, and helped to write the paper. AHuang assisted with data interpretation and helped to write the paper. AHarnden supervised HFA, assisted with study design, and helped to write the paper. All authors approved the final version of the manuscript. HFA is the guarantor.

Funding: No formal funding. HFA is an academic clinical fellow, which is a scheme partially funded by the National Institute for Health Research. The University of Oxford acted as study sponsor and had no role in the study design, data collection, analysis or interpretation, writing of the paper, or decision to submit for publication. All authors were independent from funders and sponsors.

Competing interests: All authors have completed the ICMJE uniform disclosure form at www.icmje.org/coi_disclosure.pdf (available on request from the corresponding author) and declare: no support from any organisation for the submitted work; no financial relationships with any organisations that might have an interest in the submitted work in the previous three years; no other relationships or activities that could appear to have influenced the submitted work.

Ethical approval: The study was approved by Oxford A Research Ethics Committee (reference 12/SC/0052). All participants gave informed consent before taking part.

Data sharing: Full data are available from the corresponding author on request. Consent for data sharing was not obtained, but the presented data are anonymised and the risk of identification is low. 


\section{What is already known on this topic}

Speed bumps are a commonly used traffic calming device to reduce vehicle speeds

Clinical diagnosis of acute appendicitis can be difficult, and presence of various clinical features, such as migration of pain and rebound tenderness, can be used in assessment

Some doctors routinely ask about pain on travelling over speed bumps as part of their clinical assessment, but no evidence base exists for this

\section{What this study adds}

Pain on travelling over speed bumps had a high sensitivity (97\%) but a low specificity ( $30 \%)$ for the diagnosis of appendicitis It compared favourably with other clinical features used in diagnosis of appendicitis, and therefore provides a useful addition, particularly in terms of excluding appendicitis

It may also be useful for the diagnosis of other important abdominal conditions, and its use could be extended to all presentations of the "acute abdomen"

Department for Transport. Traffic calming. Local Transport Note 01/07, 2007.

2 Towner EML, Dowswell T, Mackereth C. What works in preventing unintentional injuries in children and young adolescents? An updated systematic review. Health Developmen Agency, 2001.

3 Bunn F, Collier T, Frost C, Ker K, Roberts I, Wentz R. Area-wide traffic calming for preventing traffic related injuries. Cochrane Database Syst Rev 2003;(1):CD003110. Humes DJ, Simpson J. Acute appendicitis. BMJ 2006;333:530-4.

5 Bickell NA, Aufses AH Jr, Rojas M, Bodian C. How time affects the risk of rupture in appendicitis. J Am Coll Surg 2006;202:401-6.

6 Andersson RE. Meta-analysis of the clinical and laboratory diagnosis of appendicitis. $\mathrm{Br}$ J Surg 2004;91:28-37.

7 Walker SJ, West CR, Colmer MR. Acute appendicitis: does removal of a normal appendix matter, what is the value of diagnostic accuracy and is surgical delay important? Ann $R$ Coll Surg Engl 1995;77:358-63.

8 Lewis SR, Mahony PJ, Simpson J. Appendicitis. BMJ 2011;343:d5976.
9 Varadhan KK, Neal KR, Lobo DN. Safety and efficacy of antibiotics compared with appendicectomy for treatment of uncomplicated acute appendicitis: meta-analysis of randomised controlled trials. BMJ 2012;344:e2156.

10 Golledge J, Toms AP, Franklin IJ, Scriven MW, Galland RB. Assessment of peritonism in appendicitis. Ann R Coll Surg Engl 1996;78:11-4.

Accepted: 16 November 2012

\section{Cite this as: BMJ 2012;345:e8012}

This is an open-access article distributed under the terms of the Creative Commons Attribution Non-commercial License, which permits use, distribution, and reproduction in any medium, provided the original work is properly cited, the use is non commercial and is otherwise in compliance with the license. See: http://creativecommons.org/licenses/by$\mathrm{nc} / 2.0 /$ and http://creativecommons.org/licenses/by-nc/2.0/legalcode. 


\section{Tables}

Table 1/ Pain over speed bumps in relation to appendicitis

Appendicitis

Pain over speed bumps Positive Negative Total

\begin{tabular}{lccc} 
Positive & 33 & 21 & 54 \\
\hline Negative & 1 & 9 & 10 \\
\hline Total & 34 & 30 & 64
\end{tabular}


Table 2| Diagnostic performance (with 95\% Cl) of pain over speed bumps compared with other clinical diagnostic variables for appendicitis

\begin{tabular}{|c|c|c|c|c|c|c|}
\hline Diagnostic variable & Sensitivity (\%) & Specificity (\%) & $\begin{array}{l}\text { Positive predictive } \\
\text { value }(\%)\end{array}$ & $\begin{array}{c}\text { Negative predictive } \\
\text { value }(\%)\end{array}$ & $\begin{array}{l}\text { Positive likelihood } \\
\text { ratio }\end{array}$ & $\begin{array}{c}\text { Negative likelihood } \\
\text { ratio }\end{array}$ \\
\hline Pain over speed bumps & 97 (85 to 100) & 30 (15 to 49$)$ & 61 (47 to 74$)$ & 90 (56 to 100$)$ & $1.4(1.1$ to 1.8$)$ & $0.1(0.0$ to 0.7$)$ \\
\hline Migratory pain & 65 (46 to 80$)$ & 33 (17 to 53$)$ & 52 (36 to 68$)$ & 45 (24 to 68$)$ & $1.0(0.7$ to 1.4$)$ & $1.1(0.5$ to 2.1$)$ \\
\hline Nausea or vomiting & 79 (62 to 91$)$ & 17 (5.6 to 35$)$ & 52 (38 to 66$)$ & 42 (15 to 72$)$ & $1.0(0.8$ to 1.2$)$ & $1.2(0.4$ to 3.5$)$ \\
\hline Rebound tenderness & 71 (53 to 85$)$ & 50 (31 to 69$)$ & 62 (45 to 77$)$ & 60 (39 to 79$)$ & $1.4(0.9$ to 2.2$)$ & $0.6(0.3$ to 1.1$)$ \\
\hline
\end{tabular}


Figure

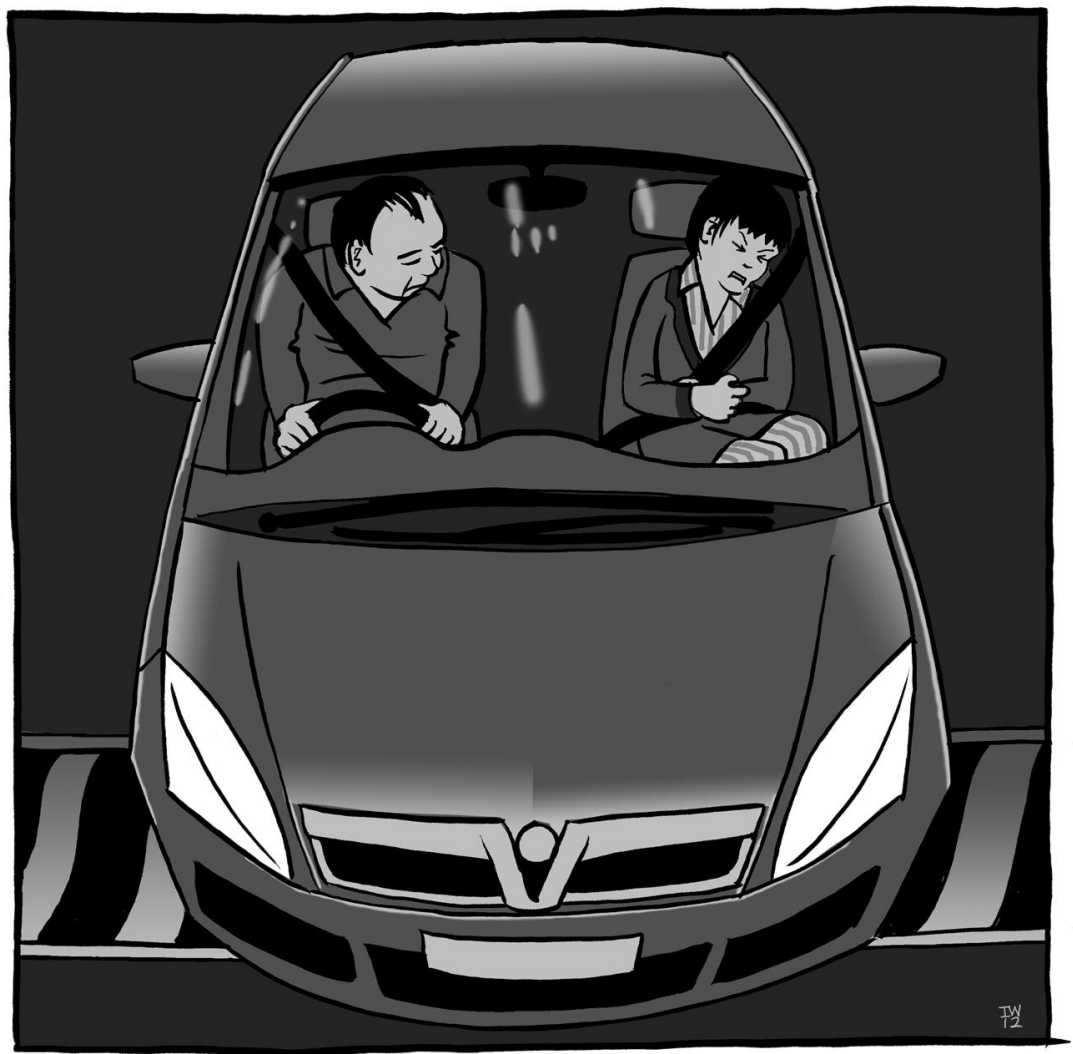

[Image: lan Williams] 